\title{
On the correlation between tensile strength and stress wave velocities of dry coherent snow based on its structural model
}

\author{
Vladimir N. Golubev, ${ }^{1}$ Anatoly D. Frolov ${ }^{2}$ \\ ${ }^{1}$ Faculty of Geography, Moscow State University, Vorobiery Gory, 119899 Moscow, Russia \\ ${ }^{2}$ Scientific Council on Earth Cryology, Russian Academy of Sciences, 11 Fersman Street, 117312 Moscow, Russia
}

\begin{abstract}
The various mechanical properties of dry coherent snow are determined by the same structural peculiarities of this medium, that can be described using the model of regular packed grains connected by rigid bonds. Analytic expressions for the many important snow mechanical parameters (density, elastic moduli, stress wave velocities, strength, etc.) are derived using the model by introducing three non-dimensional principal structural factors: texture friability, bond rigidity and coordination number. Analytic expressions that relate tensile strength to $\mathrm{P}$ and $\mathrm{S}$ stress wave velocities are proposed and used to examine the interrelations between the internal structure and tensile strength for snow-ice formations in a wide density range. The theoretically derived results and available experimental data are well correlated and form the basis for the development of non-destructive testing methods to evaluate the strength characteristics of snow, using seismic and acoustic measurements.
\end{abstract}

\section{INTRODUCTION}

Forecasting the time and place of snow avalanches on mountain slopes requires a knowledge of the average snow mechanical characteristics over the avalanche catchment area, along with data on variations in the characteristics of the snow for different layers and landforms (Golubev, 1987). Available experimental data represent the mechanical properties of snow in terms of its density as the most certain determining property. But the density is not the best comparative parameter to estimate mechanical properties of the snow. Consequently, the mechanical properties of snow referenced to density have a large spread in data. If the average grain-size is also taken into account, the variations in values for the mechanical properties decrease. Even in this case, however, snow tensile strength tests conducted at the same density may vary considerably in strength magnitude (Golubev and others, 1982).

The ambiguity in determining snow strength may be reduced by using several parameters of the snow structure including coordination number of the grain packing, thickness of bonds between the grains, etc. To obtain reliable data on these parameters, it is essential that the snow sample volume be large compared to microscale variations of $\sim 10^{4}$ interconnected grains (volume $\sim 2-3 \mathrm{~cm}^{3}$ ). Direct observation of the structural parameters and their evolution during the process of snow densification and diagenesis is extremely time-consuming, complicated and cannot cover every possible state of the snow. Therefore, it is important to develop realistic models of the relationship between snow structure and mechanical properties.

The model should be based on well-defined structural factors that make it possible to analytically estimate the principal mechanical properties of the snow and their changes during densification. This can be done by treating the ice matrix of snow as a spatial regular lattice with points formed by ice grains connected by rigid ice bonds (Golubev and Frolov, 1998, 2000). The five critical densities (including two new ones in friable snow at $\sim 140$ and $\sim 330 \mathrm{~kg} \mathrm{~m}^{-3}$ ) are found in various experiments (see Frolov, 1998) which characterize changes in the dry-snow compaction mechanisms from light fresh snow to ice. According to our model, the critical densities correspond to changes in the dominant coordination number of the grain packing from 3 to 8-10 (Golubev and Frolov, 1998). Recently published snow-hardness (Takeuchi and others, 1998) and snow-compression viscosity data (Kominami and others, 1998) confirm the existence of critical densities in friable snow established by Voronkov and Frolov (1992) and Frolov and Fedyukin (1996).

Because direct measurements of snow strength are very time-consuming and show large scatter in their values (especially in the case of light snow), it seems reasonable to develop indirect methods for strength evaluation. Such methods have the added advantage of being non-destructive and increasing the volume of tested snow and reducing the scatter of results. Usually they are seismic-acoustic techniques (see, e.g., Frolov, 1998) based on the determination of stress wave velocities in the medium and on the use of empirical relationships between the velocities and strength characteristics. In the case of snow, it appeared almost impossible to find relationships of this kind from experimental results obtained within a wide range of snow-structure characteristics. Hence, we attempt to find these relationships using our model. The model is tested by comparing our analytical results with available experimental data.

This paper presents analytical expressions for the evaluation of the tensile strength of dry coherent snow from a knowledge of stress wave velocity. The interrelations between them form a basis for a seismic-acoustic method to estimate the in situ strength characteristics of snow.

\section{BACKGROUND}

At a fixed temperature, the mechanical properties of dry coherent snow (including its strength, moduli of elasticity, 


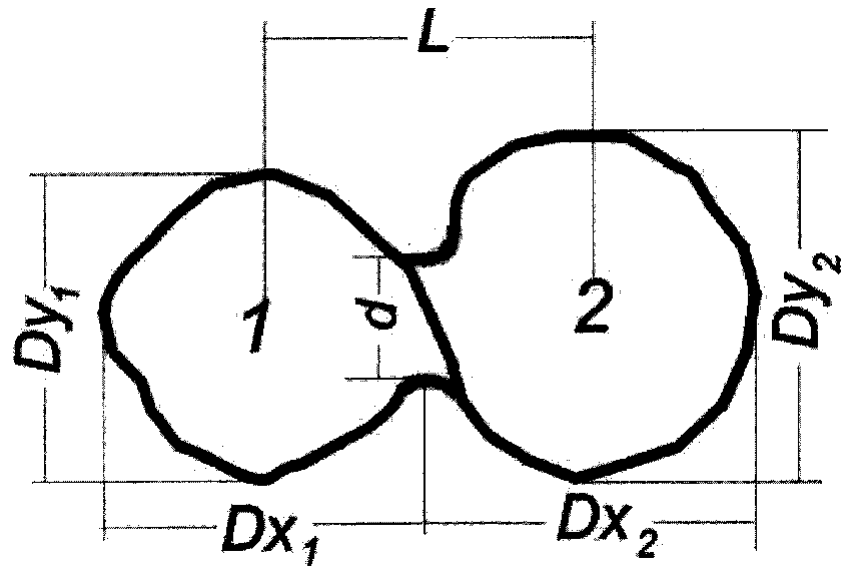

Fig. 1. Schematic presentation of contacting grains.

etc.) may be described in terms of its structure. To describe possible changes in the snow structure and mechanical properties during the process of densification and diagenesis, our model of regular grain packing (MRGP) takes into consideration averaged diameters of grains $D$ and those of rigid bonds $d$, grain center-to-center distances $L$ (Fig. 1) and the coordination number of grain packing $j$ (i.e. average number of bonds per grain). The first three parameters determine the non-dimensional rigidity factor $b=d / D$ and friability factor $k=L / D$ for the ice-matrix structure of snow. In the case of the proper contacting polyhedrons (cube, hexagonal prism, dodecahedron, rhombododecahedron), relationships between $k$ and $b$ undergo regular changes depending on their shape and coordination number. In accordance with the basic concept of our model, it is possible to express the density, moduli of elasticity and other properties as a functions of $k, b, j$ and the corresponding properties of solid ice $\left(\rho=917 \mathrm{~kg} \mathrm{~m}^{-3}\right)$ (Golubev and Frolov 1998, 2000).

In terms of our model, the snow tensile strength $\sigma_{\mathrm{s}}^{*}$ may be described as:

$\sigma_{\mathrm{s}}^{*}=\sigma_{\mathrm{i}}^{*} \frac{\rho_{\mathrm{s}}}{\rho_{\mathrm{i}}} \frac{k b^{2} j \cos a}{4 \zeta}\left\{1+\frac{j}{2}\left[1.5 k b^{2}+\left(1-b^{2}\right)^{3 / 2}-1\right]\right\}^{-1}$.

Using the equation of snow density

$$
\rho_{\mathrm{s}}=\rho_{\mathrm{i}} A \frac{\pi}{6} \frac{1}{k^{3}}\left\{1+\frac{j}{2}\left[1.5 k b^{2}+\left(1-b^{2}\right)^{3 / 2}-1\right]\right\},
$$

Equation (1) may be rewritten as follows:

$$
\sigma_{\mathrm{s}}^{*}=\sigma_{\mathrm{i}}^{*} \frac{b^{2}}{k^{2}} j \frac{A \pi j \cos a}{24 \zeta},
$$

where $\sigma_{\mathrm{i}}$ is the tensile strength of solid ice at a fixed temperature, $\rho_{\mathrm{s}}$ and $\rho_{\mathrm{i}}$ are densities of snow and solid ice, $\alpha$ is the angle of average deviation of the snow-structure elements (connecting lines between grains) from regular packing axes of symmetry, which correspond to deformation direction in our model, $\zeta$ is a parameter derived from the space grain arrangement and characterizes the number of grains per unit volume, and $A$ is a parameter determined by coordination number (Golubev and Frolov, 1998). The values of $\alpha, \zeta$ and $A$ for various $j$ values, and the ranges and median values of structure factors $k, b$ and $j$ in snow-ice formations with a range of densities are presented in Tables 1 and 2, respectively.

The expression in square brackets in Equations (1) and
Table 1. The values of snow grain-packing parameters

\begin{tabular}{rrrl}
\hline$j$ & $\alpha$ & $\zeta$ & $A$ \\
& $\circ$ & & \\
\hline 3 & 30 & 1.33 & 0.28 \\
4 & 35 & 1.00 & 0.66 \\
6 & 0 & 1.00 & 1.00 \\
8 & 0 & 1.00 & 1.16 \\
12 & 0 & 1.00 & 1.41 \\
\hline
\end{tabular}

Table 2. The intervals and average values of structure factors for snow, firn and ice

\begin{tabular}{cccc}
\hline$\rho_{\mathrm{s}}$ & $j$ & $b$ & $k$ \\
$\mathrm{~kg} \mathrm{~m}^{-3}$ & & & \\
& & & \\
\hline 100 & $3-3.5(3.1)$ & $0.03-0.2(0.07)$ & $1.15-1.35(1.25)$ \\
200 & $3-4(3.3)$ & $0.10-0.35(0.2)$ & $1.05-1.3(1.1)$ \\
300 & $3.2-5(3.6)$ & $0.15-0.40(0.27)$ & $1.00-1.15(1.05)$ \\
400 & $3.5-5.5(4.5)$ & $0.20-0.45(0.37)$ & $0.95-1.10(1.00)$ \\
500 & $4.5-7.5(6.3)$ & $0.25-0.50(0.45)$ & $0.93-1.02(0.98)$ \\
600 & $6-10(8.5)$ & $0.30-0.50(0.48)$ & $0.91-1.00(0.96)$ \\
700 & $8-11(10)$ & $0.40-0.55(0.51)$ & $0.90-0.98(0.94)$ \\
800 & $10-12(11.5)$ & $0.45-0.60(0.55)$ & $0.90-0.96(0.92)$ \\
917 & $11-13(12)$ & $0.55-0.60(0.58)$ & $0.90-0.94(0.91)$ \\
& & & \\
\hline
\end{tabular}

(2) multiplied by $j / 2$ characterizes the contribution of bonds to snow density, which is usually very small relative to unity. At $k<1.2$ and $b<0.3$ the value of this expression contributes $<5 \%$ of the density and may be omitted for snow with densities of $80-550 \mathrm{~kg} \mathrm{~m}^{-3}$. Comparison of Equations (2) and (3) reveals the different structural factors that control snow densification and strength. The snow strength depends primarily on the bond rigidity factor $b$ and on the coordination number of structure $j$; it may change 25 times within the possible range of $b=0.1-0.5$, and $>6$ times within the possible range of $j=3-6$. At the same time, changes in the snow density depend primarily (by $95 \%$ ) on variation of the structure friability factor $k$ which is cubed in Equation (2), and also on the grain-packing parameter $A$ changed from 0.28 to 1.0 .

Next we may express the Young's modulus $E_{\mathrm{s}}$ and Poisson's ratio $\nu_{\mathrm{S}}$, and consequently $\mathrm{P}$ and $\mathrm{S}$ wave velocities in the snow $\left(V_{\mathrm{ps}}\right.$ and $\left.V_{\mathrm{ss}}\right)$, in terms of snow-matrix structure factors $k$ and $b$ and parameters $\alpha, \zeta, A$ as follows:

$$
\begin{gathered}
E_{\mathrm{s}}=E_{\mathrm{i}} \frac{A \pi \cos a}{4 k\left(1+1.72 \sin ^{2} a\right)} \frac{\sqrt{1-b^{2}}}{\ln \left(1 / b^{2}\right)} \\
\cdot\left[1+\frac{(k-1)}{b^{2}} \frac{\ln \left(1 / b^{2}\right)}{\sqrt{1-b^{2}}}\right]^{-1}, \\
\nu_{\mathrm{s}}=\nu_{\mathrm{i}} k c\left[1+\frac{(k-1)}{b^{2}} \frac{\sqrt{1-b^{2}}}{\ln \left(1 / b^{2}\right)}\right]^{-1}
\end{gathered}
$$

and

$V_{\mathrm{ps}}=0.9 V_{\mathrm{pi}}$

$\sqrt{\frac{\cos a}{\left(1+1.72 \sin ^{2} \alpha\right)} \frac{k^{2} \sqrt{1-b^{2}}}{Z \ln \left(1 / b^{2}\right)} \frac{\left(1-\nu_{\mathrm{i}} k c / z\right)}{\left(1-\nu_{\mathrm{i}} k c / Z\right)\left(1-2 \nu_{\mathrm{i}} k c / Z\right)}}$, 
Table 3. Ratio of respective maximum and minimum values of averaged experimental data on velocities and dynamic elastic moduli for different stages of snow-ice formation texture ordering within given density ranges

\begin{tabular}{|c|c|c|c|c|c|c|}
\hline & \multicolumn{6}{|c|}{ jvalue } \\
\hline & & 3 & 4 & 6 & 8 & $10-12$ \\
\hline$\rho\left(\mathrm{kg} \mathrm{m}^{-3}\right)$ & $50-140$ & $140-330$ & $330-540$ & $540-730$ & $730-840$ & $>840$ \\
\hline$V_{\mathrm{p}}$ & 2.9 & 2.3 & 3.9 & 1.45 & 1.12 & 1.10 \\
\hline$V_{\mathrm{s}}^{\mathrm{P}}$ & 2.7 & 2.1 & 3.7 & 1.39 & 1.13 & 1.08 \\
\hline$E$ (Young's) & 21.0 & 11.0 & 23.0 & 2.70 & 1.35 & 1.25 \\
\hline$K$ (bulk) & 24.0 & 16.0 & 29.0 & 8/3.15 & 1.52 & 1.40 \\
\hline$\mu$ (shear) & 21.0 & 10.0 & 22.0 & 2.64 & 1.34 & 1.24 \\
\hline$\nu$ (Poisson's) & 6.0 & 2.1 & 1.09 & 1.12 & $\sim 1.08$ & $\sim 1.09$ \\
\hline
\end{tabular}

$V_{\mathrm{ss}}=5.15 V_{\mathrm{si}} \sqrt{\frac{\cos \alpha}{\left(1+1.72 \sin ^{2} \alpha\right)} \frac{k^{2} \sqrt{1-b^{2}}}{Z \ln \left(1 / b^{2}\right)} \frac{1}{2\left(1+\nu_{\mathrm{i}} k c / Z\right)}}$,

where

$$
Z=1+\frac{k-1}{b^{2}} \frac{\sqrt{1-b^{2}}}{\ln \left(1 / b^{2}\right)} .
$$

In Equations (4-7), $E_{\mathrm{i}}, \nu_{\mathrm{i}}, V_{\mathrm{pi}}, V_{\mathrm{si}}$ are Young's modulus, Poisson's ratio, velocities of $\mathrm{P}$ and $\mathrm{S}$ stress waves of ice, respectively. The lateral deformation of the medium as a function of coordination number $j$ is given by $c$ : that is, $c=(j-2) / 4$ at $j<6$, and $c=1$ at $j \geq 6$.

In the process of the snow densification, the coordination number $j$ increases along with the ordering and stability of the ice-matrix structure. Dominance of one or another coordination number may be characterized by a certain range (interval) of the snow density. Every such interval is characterized by a certain mechanism and regularity of the compaction process (Golubev and Frolov, 2000), while a change-over from one mechanism to another occurs at the boundaries of the interval, near the critical densities 140, 330, 540, 710, $830 \mathrm{~kg} \mathrm{~m}^{-3}$. Changes in $\mathrm{P}$ and $\mathrm{S}$ stress wave velocities and in dynamic elastic moduli $(E, K, \mu$ and $\nu)$ of the snow-ice formations vary distinctly at different stages of the structure ordering (Table 3) (Frolov, 1998).

The pronounced change (increase) in all the parameters occurs during the process of snow densification firstly when the density increases from $\rho \cong 50$ to $140 \mathrm{~kg} \mathrm{~m}^{-3}$. On further compaction $\left(\rho \approx 140-330 \mathrm{~kg} \mathrm{~m}^{-3}\right)$ the magnitude of increase in the parameters is considerably reduced. Then (except for Poisson's ratio) it grows noticeably once again, but when $j>6$ (which corresponds to firn and ice) the magnitude of increase in mechanical parameters of the snow-ice medium is only lessened. One may well suppose a similar pattern in the strength changes.

These data are indicative of different compaction mechanisms acting at various stages of dry-snow densification (grain displacement into pore space, deformation of the ice-matrix space lattice, ice grain deformation, etc.). These densification stages must be taken into account when determining the most reliable relationships between structural parameters and mechanical properties (Golubev and Frolov, 2000).

\section{RESULTS AND DISGUSSION}

We calculated the tensile strength and velocities of $\mathrm{P}$ and $\mathrm{S}$
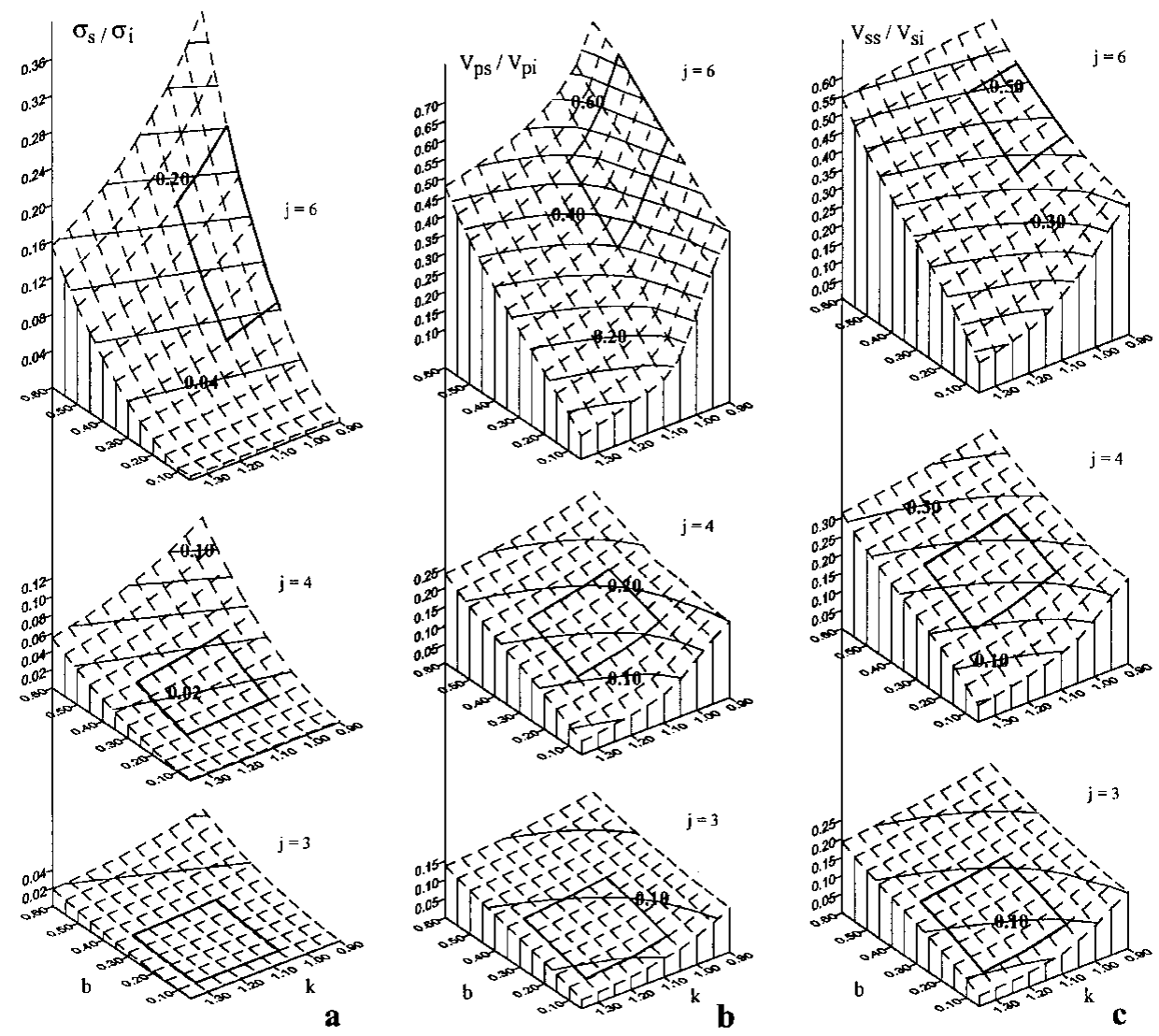

Fig. 2. Calculated relative snow tensile strength ( a), relative velocities of $P$ waves $(b)$ and $S$ waves $(c)$ in snow vs structural indexes $b$ and $k$. 


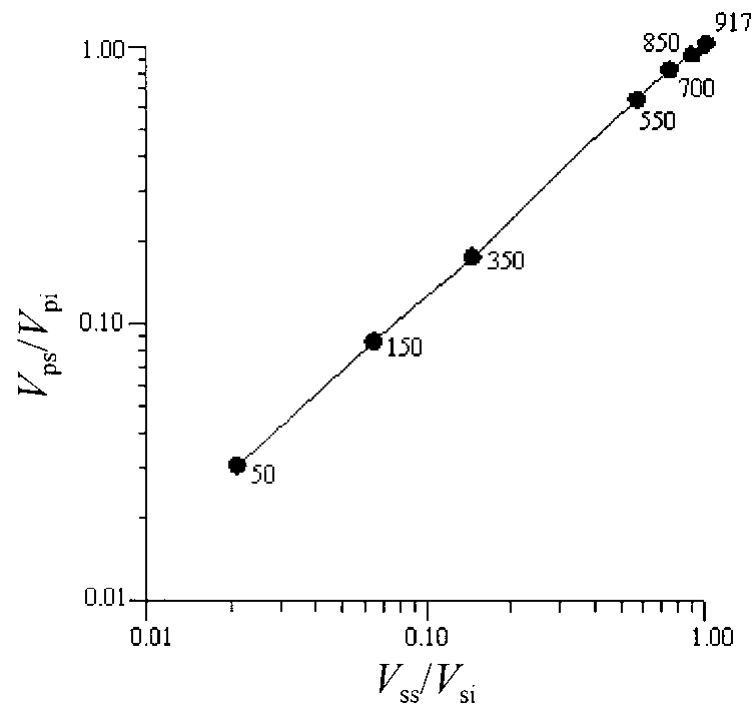

Fig. 3. Relationship between averaged relative velocities of $P$ and $S$ stress waves for snow-ice formations. Points on graph are the densities in $\mathrm{kg} \mathrm{m}^{-3}$.

stress waves (relative to solid ice) for snow of various structures, using Equations (3), (6) and (7) assuming the tensile strength $\sigma_{\mathrm{i}}=1.5 \mathrm{MPa}$ and the stress wave velocities $V_{\mathrm{pi}}=$ $4 \mathrm{~km} \mathrm{~s}^{-1}, V_{\mathrm{si}}=2 \mathrm{~km} \mathrm{~s}^{-1}$. The results are presented as unified diagrams, the surfaces of which illustrate all ranges of possible values of these parameters with dominant coordination numbers $j=3,4$ and 6 , at $k=0.90-1.35$ and $b=0.05-0.6$ (Fig. 2). Moreover, on these diagrams the regions of the most probable relative magnitudes of $\sigma_{\mathrm{s}}, V_{\mathrm{ps}}$ and $V_{\mathrm{ss}}$ are marked. These regions were determined from the regularities of various possible polyhedron contacts and direct measurements on samples of real snow of different densities (Golubev, 1982; Golubev and Frolov, 1998). The regions correspond to $k=1.05-1.30$ and $b=0.1-0.35$ at $j=3\left(\rho_{\mathrm{s}}=\right.$ 80-180 $\left.\mathrm{kg} \mathrm{m}^{-3}\right)$; to $k=1.00-1.20$ and $b=0.2-0.4$ at $j=4$ $\left(\rho_{\mathrm{s}}=200-400 \mathrm{~kg} \mathrm{~m}^{-3}\right)$; to $k=0.90-1.05$ and $b=0.3-0.5$ at $j=6\left(\rho_{\mathrm{s}}=450-600 \mathrm{~kg} \mathrm{~m}^{-3}\right)$ (Fig. 2). From our calculations (Fig. 2a) it follows that the most probable calculated values of snow relative tensile strength changes are: from 0.001 to 0.01 (mean 0.004 ) at $j=3$; from 0.015 to 0.045 (mean 0.03 ) at $j=4$; and from 0.07 to 0.24 (mean 0.14 ) at $j=6$. These values are in good agreement with experimental results (e.g. Mellor, 1977; Golubev and others, 1982).

Figure $2 \mathrm{~b}$ and c illustrate the calculated changes of relative values of $\mathrm{P}$ and $\mathrm{S}$ stress wave velocities in snow with various structures. All available experimental data on these velocities obtained in real snow of different densities in Antarctica, Greenland, Khibiny and in laboratory experiments (see reviews in Mellor, 1977; Sommerfeld, 1982; Frolov, 1998) fall within the marked areas. As the $V_{\mathrm{ps}}$ and $V_{\mathrm{ss}}$ velocities are functions of the same variables $k$ and $b$ (Equations (6) and (7)), it is not surprising that there is a good correlation between these velocities for a snow medium. The relationship between relative $V_{\mathrm{ps}}$ and $V_{\mathrm{ss}}$ over the whole density range of snow-ice formations, obtained from averaged experimental data, is shown in Figure 3 . So, when $V_{\mathrm{p}}$ velocity in snow is determined by known seismic-acoustic techniques, and $V_{\mathrm{s}}$ velocity is estimated from Figure 3 , all the elasticity moduli of the snow medium in question can be calculated, and, consequently, $k$ and $b$ structural factors may be determined.

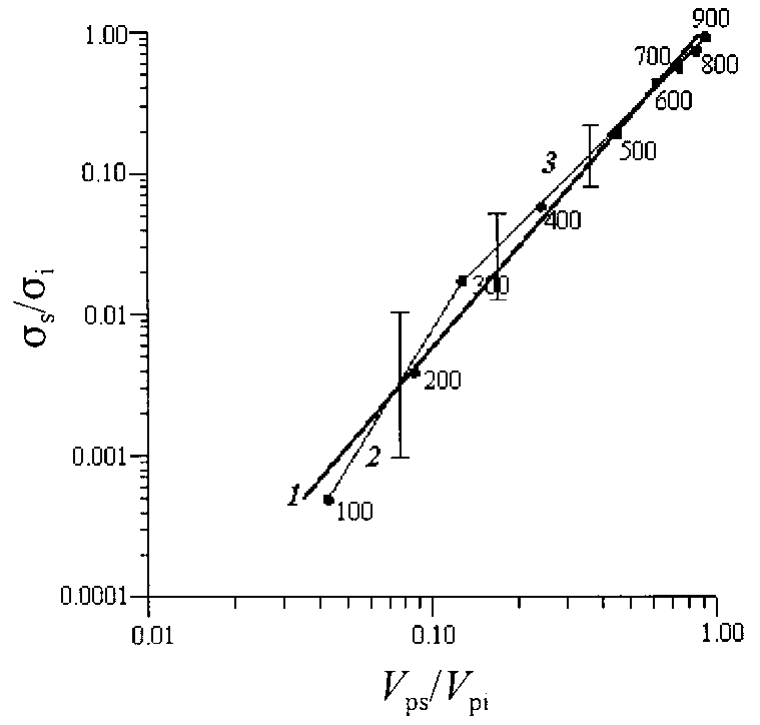

Fig. 4. Relationship between averaged calculated relative tensile strength and averaged experimental relative velocity of $P$ stress waves for snow-ice formations. Points on graph are the densities in $\mathrm{kg} \mathrm{m}^{-3}, 1,2,3$ (see text). Vertical lines show the possible scattering of data for probable values of structural factors $k$ and $b$ at $j=3,4,6$.

The principal parameters of the snow mechanical properties which control the snow-cover stability on mountain slopes are its tensile and shear strength. For isotropic snow and ice formations the shear, tensile and compression strength are related as $1: 1.5: 2$, which provides a method of estimating the complete strength characteristics for snow from any one of the strength measures. Snow tensile strength and longitudinal stress wave velocity are functions of the same parameters (Equations (3) and (6)), providing the possibility that the strength characteristics of snow can be evaluated from seismic-acoustic studies of snow cover.

Figure 4 shows the relationships between the relative snow tensile strength and the longitudinal stress wave velocity for various densities from friable snow to solid ice. The calculation of $\sigma_{\mathrm{s}} / \sigma_{\mathrm{i}}$ was carried out using Equation (3) and data from Tables 1 and 2. The corresponding values $V_{\mathrm{ps}} / V_{\mathrm{pi}}$ were taken from averaged experimental data (see Mellor, 1977; Sommerfeld, 1982; Frolov, 1998). In the density range $100-917 \mathrm{~kg} \mathrm{~m}^{-3}$ the relationship between averaged values of $\sigma_{\mathrm{s}} / \sigma_{\mathrm{i}}$ and $V_{\mathrm{ps}} / V_{\mathrm{pi}}$ is approximated by the following function (Fig. 4, curve 1):

$$
\frac{\sigma_{\mathrm{s}}}{\sigma_{\mathrm{i}}}=\sqrt{2}\left(\frac{V_{\mathrm{ps}}}{V_{\mathrm{pi}}}\right)^{2,3} .
$$

However, one can see from the graph that for friable snow $\left(\rho_{\mathrm{s}} \leq 300 \mathrm{~kg} \mathrm{~m}^{-3}\right)$ a better correlation between Equation (8) and the data occurs when the exponent is 3 (Fig. 4, curve 2), and for the density $\rho_{\mathrm{s}}>300 \mathrm{~kg} \mathrm{~m}^{-3}$ the best fit correlation occurs when the exponent decreases approximately to 2 (Fig. 4, curve 3). This decrease of the $\sigma_{\mathrm{s}}$ slope indicates the importance of changes in snow texture for strength and stress wave propagation, especially in friable snow.

\section{GONGLUDING REMARKS}

The good correlation established using our model between stress wave velocity $V_{\mathrm{ps}}$ and tensile strength $\sigma_{\mathrm{s}}$ for dry 
coherent snow-ice formations indicates that averaged snow strength may be accurately determined by the use of seismicacoustic data. Comparison with available experimental data has not revealed any important contradictions. These interrelations can be used to interpret stress wave velocity data to determine snow strength. In addition, the relationship between velocities $V_{\mathrm{p}}$ and $V_{\mathrm{s}}$ and snow structure in dry snow-ice formations provides a means of interpreting seismic-acoustic data (including well logging, pit, dug walls profiling, etc.) to estimate all elastic moduli and (when solving the inverse problem) structural factors of the snow. Our presentation of results relative to solid ice reduces the influence of temperature. Some uncertainty exists as to the possibility that properties of bulk ice (used by us) may not be equivalent to those of bond ice. The corresponding properties of bond ice are not well known.

This study, as well as our earlier work (Golubev and Frolov, 1998, 2000), demonstrates that MRGP of dry coherent snowice formations is adequate to the object and can be applied to evaluate their mechanical properties from seismic-acoustic data as a first approximation useful for the engineering targets.

\section{ACKNOWLEDGEMENTS}

We thank P. Grebennikov and D. Frolov for their contributions to the calculations and manuscript preparation. The studies described in this paper were partly funded by the Russian Foundation of Basic Researches (grant 98-05-64865).

\section{REFERENCES}

Frolov, A. D. 1998. Elektricheskiye i uprugiye svoystva merzlych porod i ldov [Electric and elastic properties of frozen earth materials]. Pushchino, RossiyskoyAkademii Nauk. Izdatel'stvo Pushchinskogo Nauchnogo Centra.

Frolov, A. D. and I.V. Fedyukin. 1996. Akusticheskiye kharakteristiki snezhnoledyanych obrazonvanii plotnost'yu $300-917 \mathrm{~kg} \mathrm{~m}^{-3}$ [Acoustic characteristics of snow-ice formation in the density range from $\left.300-917 \mathrm{~kg} \mathrm{~m}^{-3}\right]$. In Yamshchikov, V. S., ed. Problemy geoakustiki: metodika i sredstva [Problems of geoacoustics: techniques and tools]. Moscow, Russian Acoustic Society, 181-184.

Frolov, A. D. and I.V. Fedyukin. 1998. Elastic properties of snow-ice formations in their whole density range. Ann. Glaciol., 26, 55-58.

Golubev, V. N. 1982. Zavisimost' uprugikh svoystv snega ot yego struktury [Dependence of the elastic properties of the snow structure]. Mater. Glyatsiol. Issled. 44, 65-73.

Golubev, V. N. 1987. Nekotoryye zakonomernosti prostranstvennoi neodnorodnosti svoystv i strieniya snezhnogo pokrova na sklonakh gor [Some regularities of the spatial non-uniformity of snow-cover properties and structure on mountain slopes]. In Trudy II Vsesoyuznogo Soveshchaniya po Lavinam [Proceedings, 2nd All-Union Symposium on Avalanches]. Leningrad, Gidrometeoizdat, 220-228.

Golubev, V. N. and A. D. Frolov. 1998. Modelling the change in structure and mechanical properties in dry-snow densification to ice. Ann. Glaciol., 26, 45-50.

Golubev, V. N. and A. D. Frolov. 2000. Model of structure and mechanical properties of dry granular snow. Ann. Glaciol., 31, 434-438.

Golubev, V. N., V. K. Voitkovskiy and T.V. Boltneva. 1982. Vliyaniye struktury na mekhanicheskiye svoystva snega [The influence of snow structure on its mechanical properties]. Mater. Glyatsiol. Issled. 45, 109-113.

Kominami, Y., Y. Endo, S. Niwano and S. Ushioda. 1998. Viscous compression model for estimating the depth of new snow. Ann. Glaciol., 26, 77-82.

Mellor, M. 1977. Engineering properties of snow. F. Glaciol., 19(81), 15-66.

Sommerfeld, R. A. 1982. A review of snow acoustics. Rev. Geophys. Space Phys., 20(1), 62-66.

Takeuchi, Y., Y. Nohguchi, K. Kawashima and K. Izumi. 1998. Measurement of snow-hardness distribution. Ann. Glaciol., 26, 27-30.

Voronkov, O. K. and A. D. Frolov. 1992. Fizicheskiye svoystva gornykh porod pri otritsatelynykh temperaturakh [Physical properties of rocks and soils at negative temperatures]. In Dortman, N. B., ed. Petrofizika [Petrophysics]. Vol. 3. Zemnaya kora imantiya [Earth core and mantle]. Moscow, Nedra, 43-65. 